RAD Conference Proceedings, vol. 4, pp. 45-49, 2020

ISSN 2466-4626 (online) | DOI: 10.21175/RadProc.2020.09

www.rad-proceedings.org

\title{
RADIOPROTECTIVE EFFECT OF HUMAN LACTOFERRIN AGAINST GAMMA-IRRADIATION WITH SUBLETHAL DOSE
}

\author{
Marina Yu. Kopaeva ${ }^{{ }^{*}}$, Irina B. Alchinova3, Mikhail V. Nesterenko², \\ Anton B. Cherepov', Marina S. Demorzhi3, Irina Yu. Zarayskaya', Mikhail Yu. Karganov3 \\ ${ }^{1}$ National Research Center Kurchatov Institute, Moscow, Russia \\ 2"Lactobio" LLC, Moscow, Russia \\ 3Institute of General Pathology and Pathophysiology, Moscow, Russia
}

\begin{abstract}
The aim of this study was to investigate the effects of human lactoferrin ( $h L f$ ) in mice exposed to acute sublethal gamma-irradiation. $\mathrm{C} 57 \mathrm{Bl} / 6$ male mice were used for the experiments. Animals from experimental groups were exposed to whole-body gamma-radiation at a dose of $7.5 \mathrm{~Gy}$. Some animals received an intraperitoneal injection of lactoferrin (Lf) immediately and then at 24 hours after the irradiation. The effect of Lf on survival rate and life span was studied. Changes in the physiological parameters were evaluated by laser correlation spectroscopy of blood serum, histological examination of the liver, and blood leukocyte shift index count on day 30 after irradiation. The Lf administration increased the survival rate and life span of irradiated mice during the experiment. In addition, Lf had a compensatory effect on the white blood formula, serum composition and liver condition of irradiated animals.
\end{abstract}

Keywords: Human lactoferrin, acute gamma-irradiation, $\mathrm{C}_{57} \mathrm{Bl} / 6$ mice, laser correlation spectroscopy, survival rate

\section{INTRODUCTION}

The pathogenic processes induced by ionizing radiation are currently being extensively researched. The acute and late effects of radiation exposure, including therapeutic effects, are of great medical interest. Patients undergoing radiotherapy may develop immune suppression, serious pathological changes in most sensitive organs and hematopoietic system. Therefore, an active search is underway for non-toxic radioprotective compounds that can protect normal tissues from radiation damage and restoring the function of all systems.

Lactoferrin (Lf) is a multifunctional protein of the transferrin family that is widely represented in various secretory fluids of mammals. The prospect of studying the possibility of using Lf in the therapy of radiation diseases is due to its immunomodulatory and hemostimulating activities, and the capacity of reducing oxidative stress and controlling excessive inflammatory responses [1], [2]. Previously, it was shown the therapeutic effect of Lf purified from cow's milk after single whole-body X-ray irradiation [3], [4].

The aim of this research was to investigate the effects of human Lf (hLf) in mice exposed to acute gamma-irradiation at a sublethal dose.

\section{MATERIALS AND METHODS}

C57Bl/6 male mice (age 2.0-2.5 months) were obtained from the Branch of the Shemyakin and
Ovchinnikov Institute of Bioorganic Chemistry, Russian Academy of Sciences (Pushchino, Moscow Region, Russia). The animals were kept in plastic cage ( 5 mice per cage) at a $12 \mathrm{~h}$ light/dark cycle in a temperature- and humidity-controlled environment with free access to standard laboratory food and water. All experimental procedures were performed in accordance with the rules of the Ministry of Health of the Russian Federation (No. 267 of 19.06.2013) and the Local Ethics Committee for Biomedical Research of the National Research Center Kurchatov Institute (protocol No. 1 of 13.02.2020). Human Lf was isolated from the colostrum by preparative ion-exchange chromatography [5] followed by purification with affinity heparin-sepharose sorbent [6].

Animals were randomly divided into 3 experimental (IR, IR+Lf, IR+Lfx2; $n=17$ per group), 3 control (Cont, Cont+Lf, Cont+Lfx2; $n=8$ per group), and intact $(n=7)$ groups. Mice from experimental groups were exposed to whole-body gamma-radiation from a cobalt-60 $\left({ }^{60} \mathrm{Co}\right)$ source at $7.5 \mathrm{~Gy}$ (at a dose rate of $0.6 \mathrm{~Gy} / \mathrm{min}$ ). The control mice were sham irradiated. Human Lf was dissolved in $0.4 \mathrm{ml}$ of saline to adjust the concentration to $4.0 \mathrm{mg} /$ animal. Some animals received an intraperitoneal injection of Lf immediately (IR+Lf, IR+Lfx2, Cont+Lf, Cont+Lfx2 groups) and then at 24 hours (IR+Lfx2, Cont+Lfx2 groups) after the treatments. The animals of IR and Cont groups received $0.9 \%$ physiological saline twice. Dosage of protein was based on the results of our previous experiments [7], [8].

\footnotetext{
"m.kopaeva@mail.ru
} 
M. Yu. Kopaeva et al., Radioprotective effect of human lactoferrin..., RAD Conf. Proc., vol. 4, 2020, 45-49

The body weights of the mice were measured every three days after $2 \mathrm{~h}$ food deprivation. The survival rate and life span were observed for 30 days. Changes in the physiological parameters were evaluated by laser correlation spectroscopy of blood serum (LCS), histological examination of the liver, and blood leukocyte shift index count on day 30 after irradiation. The mice were decapitated under deep isoflurane anesthesia, and the blood was collected. The serum was obtained from the whole mouse blood. The samples were left to stay for $2 \mathrm{~h}$ at $4^{\circ} \mathrm{C}$, centrifuged at $2000 \mathrm{RCF}$ for $10 \mathrm{~min}$, and stored at $-20^{\circ} \mathrm{C}$ until analysis. Measurements were carried out on a LKS-O3 laser correlation spectrophotometer (INTOKS) after the samples were thawed at room temperature and diluted with a physiological saline in a ratio of 1:10 [9].

Changes in the blood were assessed by differential analysis of blood smears after Pappenheim staining (combined May-Grunwald-Giemsa stain). Index of blood leukocyte shift (IBLS) was determined by the following formula: $I B L S=$ (eosinophils + basophils + neutrophils) / (monocytes + lymphocytes). The values of the formed blood elements were taken as a percentage.

The liver was isolated to assess morphological changes caused by the damaging effects of radiation. The detailed method was the same as described previously [10], 4\% neutral formalin solution was used as a fixative. Tissue sections $(5 \mu \mathrm{m})$ were stained by hematoxylin and eosin (H\&E) using the standard staining procedure and were examined using a light microscope.

The statistical analysis was performed using GraphPad Prizm 6.01 software (La Jolla, CA, USA). The normality of data distribution was assessed with the Shapiro-Wilk test. The nonparametric KruskalWallis ANOVA with post hoc Dunn's test for multiple comparisons was employed. The survival rate of mice was analyzed by the Kaplan-Meier method (GehanBreslow-Wilcoxon test). $\mathrm{P}$ values < 0.05 were considered to be significant.

\section{RESULTS AND DISCUSSION}

The first mouse deaths occurred on days 8,11 , and 12 after irradiation in the groups IR, IR+Lf and IR+Lfx2, respectively. The $\mathrm{Lf}$ administration increased the survival rate of irradiated mice from $29.4 \%$ (IR group) to $76.5 \%$ (IR+Lfx2, $\mathrm{p}=0.003$ ) and $70.6 \%$ (IR+Lf, $\mathrm{p}=0.010)$. The protein treatment also significantly increased the life span of irradiated mice during the experiment (IR+Lfx2: $26.1 \pm 1.8$; and IR+Lf: $24.9 \pm 2.0$ compared to IR: $16.5 \pm 2.3$, days; $\mathrm{p} 1=0.003, \mathrm{p} 2=0.010$, respectively) [8]. These data are consistent with the results obtained earlier. Effect of bovine Lf after single whole body X-ray irradiation of mice [3] and Lf obtained by biotechnological method from rabbit colostrum after total $\gamma$-irradiation of guinea-pigs [11] was shown in increasing the survival rate of animals.

On days 9 and 12 after irradiation, the body weight of animals from all experimental groups decreased and significantly differed from the body weight of control animals (Table 1). The normal level of this parameter was not recovered in IR group even by the 3oth day. Whereas the mice weights in both Lf treatment irradiated groups were restored to the level of the corresponding control on days 15 (IR+Lfx2) and 18 (IR+Lf), respectively. It has been previously reported that the body weights of the mice decreased between day 8 and 10 after $\mathrm{X}^{-}$ray irradiation at 7.0 Gy [4]. Between days 20 and 30 , the body weight was significantly higher in the animals received a diet containing bovine Lf. The mice were fed for 7 days prior to irradiation and for 30 continuous days following irradiation.

Table 1. Body weights ( $\mathrm{g}$ ) of irradiated mice at different time points

\begin{tabular}{|c|c|c|c|c|c|c|c|c|c|c|c|c|c|c|}
\hline \multirow{3}{*}{$\begin{array}{l}\text { Time after } \\
\text { irradiation, } \\
\text { days }\end{array}$} & \multicolumn{14}{|c|}{ Groups } \\
\hline & \multicolumn{2}{|l|}{ Cont } & \multicolumn{2}{|c|}{ Cont + Lf } & \multicolumn{2}{|c|}{ Cont+Lfx2 } & \multicolumn{2}{|l|}{ IR } & \multicolumn{2}{|l|}{$\mathrm{IR}+\mathrm{Lf}$} & \multicolumn{2}{|c|}{ IR+Lfx2 } & \multicolumn{2}{|c|}{ Intact } \\
\hline & $\mathrm{M} \pm \mathrm{SE}$ & $\mathrm{n}$ & $\mathrm{M} \pm \mathrm{SE}$ & $\mathrm{n}$ & $\mathrm{M} \pm \mathrm{SE}$ & $\mathrm{n}$ & $\mathrm{M} \pm \mathrm{SE}$ & $\mathrm{n}$ & $\mathrm{M} \pm \mathrm{SE}$ & $\mathrm{n}$ & $\mathrm{M} \pm \mathrm{SE}$ & $\mathrm{n}$ & $\mathrm{M} \pm \mathrm{SE}$ & $\mathrm{n}$ \\
\hline-1 & $23.4 \pm 0.6$ & 8 & $23.6 \pm 0.7$ & 8 & $23.2 \pm 0.7$ & 8 & $23.1 \pm 0.5$ & 17 & $23.1 \pm 0.6$ & 17 & $23.2 \pm 0.5$ & 17 & $23.4 \pm 0.8$ & 7 \\
\hline 3 & $23.5 \pm 0.5$ & 8 & $23.9 \pm 0.7$ & 8 & $23.6 \pm 0.7$ & 8 & $22.7 \pm 0.5$ & 17 & $22.3 \pm 0.6$ & 17 & $23.0 \pm 0.4$ & 17 & $23.5 \pm 0.9$ & 7 \\
\hline 6 & $24.3 \pm 0.6$ & 8 & $24.6 \pm 0.6$ & 8 & $24.1 \pm 0.8$ & 8 & $22.2 \pm 0.5^{*}$ & 17 & $22.2 \pm 0.6^{*}$ & 17 & $22.6 \pm 0.5$ & 17 & $24.1 \pm 0.8$ & 7 \\
\hline 9 & $24.3 \pm 0.5$ & 8 & $24.6 \pm 0.7$ & 8 & $24.7 \pm 0.7$ & 8 & $22.1 \pm 0.4^{*}$ & 14 & $22.1 \pm 0.6^{*}$ & 17 & $22.6 \pm 0.5^{*}$ & 17 & $24.4 \pm 0.8$ & 7 \\
\hline 12 & $24.9 \pm 0.6$ & 8 & $25 \cdot 3 \pm 0.8$ & 8 & $25.1 \pm 0.6$ & 8 & $21.4 \pm 0.6^{* *}$ & 9 & $22.2 \pm 0.7^{*}$ & 15 & $22.9 \pm 0.6^{*}$ & 15 & $24.8 \pm 0.8$ & 7 \\
\hline 15 & $25.2 \pm 0.5$ & 8 & $25.3 \pm 0.7$ & 8 & $25.1 \pm 0.6$ & 8 & $21.1 \pm 1.1^{* *}$ & 7 & $21.8 \pm 0.9^{*}$ & 13 & $22.8 \pm 0.8$ & 14 & $25.1 \pm 0.9$ & 7 \\
\hline 18 & $25.3 \pm 0.5$ & 8 & $25.2 \pm 0.8$ & 8 & $25.5 \pm 0.6$ & 8 & $21.5 \pm 1.2^{*}$ & 6 & $22.5 \pm 1.1$ & 12 & $23.4 \pm 0.8$ & 13 & $25.4 \pm 0.9$ & 7 \\
\hline 21 & $25.2 \pm 0.6$ & 8 & $25.5 \pm 0.8$ & 8 & $25.5 \pm 0.7$ & 8 & $22.4 \pm 1.2^{*}$ & 5 & $23.0 \pm 1.0$ & 12 & $24.0 \pm 0.8$ & 13 & $25.2 \pm 1.0$ & 7 \\
\hline 24 & $25.8 \pm 0.5$ & 8 & $25.6 \pm 0.8$ & 8 & $26.3 \pm 0.6$ & 8 & $22.2 \pm 1.2^{*}$ & 5 & $23.4 \pm 1.0$ & 12 & $25.0 \pm 0.7$ & 13 & $25.8 \pm 1.1$ & 7 \\
\hline 27 & $26.1 \pm 0.6$ & 8 & $26.1 \pm 0.9$ & 8 & $26.4 \pm 0.6$ & 8 & $22.6 \pm 1.3^{*}$ & 5 & $24.1 \pm 0.9$ & 12 & $25 \cdot 3 \pm 0.6$ & 13 & $25.8 \pm 1.1$ & 7 \\
\hline 30 & $26.2 \pm 0.6$ & 8 & $26.2 \pm 0.9$ & 8 & $26.9 \pm 0.6$ & 8 & $23.2 \pm 1.4^{*}$ & 5 & $24.5 \pm 0.8$ & 12 & $25.7 \pm 0.6$ & 13 & $26.0 \pm 1.0$ & 7 \\
\hline
\end{tabular}

The values are given as mean \pm standard error of the mean. ${ }^{*} \mathrm{p}<0.05,{ }^{* *} \mathrm{p}<0.005$ compared to the corresponding control group at an each time point (Kruskal-Wallis ANOVA followed by post hoc Dunn's multiple comparisons test). IR, irradiated mice; IR+Lf, irradiated mice received Lf; IR+Lfx2, irradiated mice received Lf twice; n, number of animals in the group at an each time point. 
Laser correlation spectroscopy (LCS) based on measurement of spectral characteristics of light scattering allows measuring particle size in native biological fluids. Irradiation caused a significant increase in the contribution to light scattering of particles from 165.5 to $404.7 \mathrm{~nm}$ in size compared to the control group, which is associated with the development of the inflammatory process as a reaction to cell damage [12]. The results of post hoc analysis are shown in Figure 1. Lf injection led to a shift in the curves to the control values, which may indicate the restoration of serum homeostasis. The values in the Cont, Cont+Lf, and IR+Lf groups did not differ significantly from each other (Figure 1A). Double administration of Lf to control animals caused an increase in the contribution to light scattering of particles with a radius of $20.6-37.43 \mathrm{~nm}$, which is probably associated with an increase in the concentration of free Lf in the blood (Figure 1B).
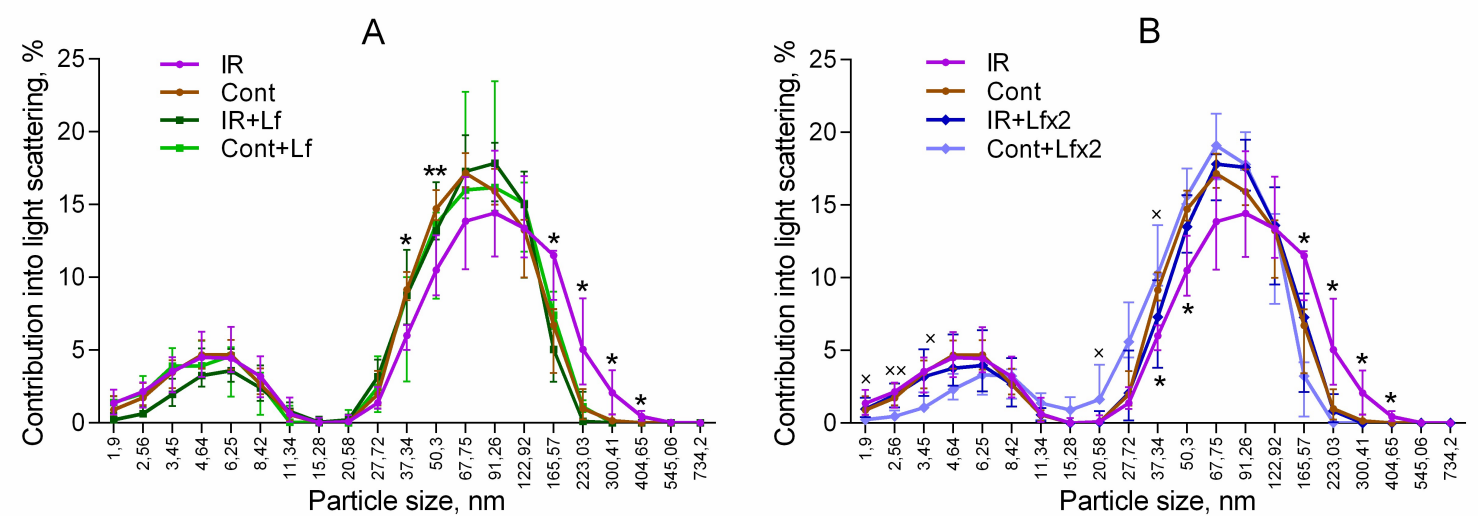

Figure 1. Changes in LC spectra of mouse blood serum. ${ }^{*} \mathrm{p}<0.05,{ }^{* *} \mathrm{p}<0.01$ IR compared to the Cont group; $\mathrm{x}_{\mathrm{p}}<0.05,{ }^{\mathrm{xx}} \mathrm{p}<0.01 \mathrm{IR}+\mathrm{Lfx} 2$ compared to the Cont+Lfx2 group at an each time point (Kruskal-Wallis ANOVA followed by post hoc Dunn's multiple comparisons test). Values are presented as median \pm interquartile range

Changes in the composition of peripheral blood are one of the main criteria for evaluating the effect of radiation on the body. The acute gamma-irradiation induced an increase of IBLS value in our model. The IR group exhibited a significant increase in this parameter compared to the Cont group on day $30[\mathrm{H}(5, \mathrm{~N}=34)=$ 12.28, $\mathrm{p}=0.0311$; post hoc $\mathrm{p}=0.0077$ ] (Figure 2). This is consistent with published data [12].

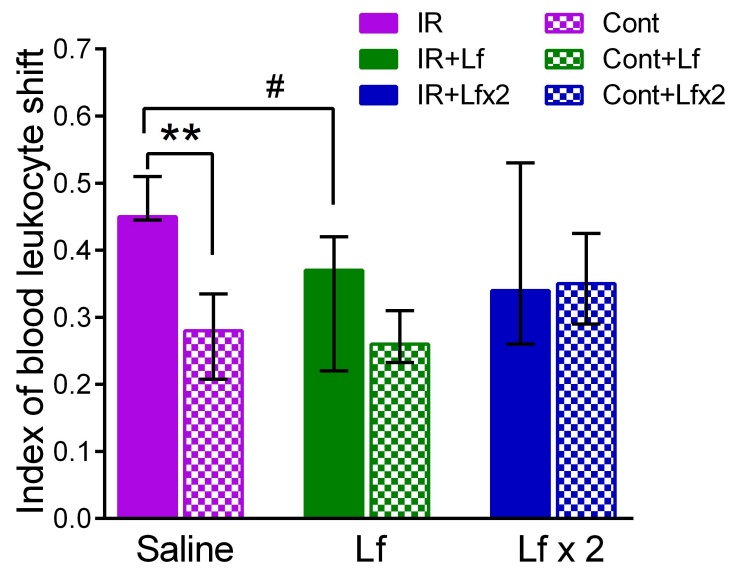

Figure 2. Index of mouse blood leukocyte shift on day 30 after irradiation. **p $<0.01$ IR compared to the Cont group; \#p $<0.05$ IR compared to the IR+Lf group (Kruskal-Wallis ANOVA followed by post hoc Dunn's multiple comparisons test). Values are presented as median \pm interquartile range
An increase in IBLS characterizes the presence of an inflammatory reaction and a disturbance of the body's immunological reactivity. Administration of Lf to the control animals (Cont+Lf and Cont+Lfx2) did not affect the value of IBLS. The present data showed that the Lf-treated irradiated mice did not differ from the corresponding controls in IBLS ( $\mathrm{p}=$ 0.868 for IR+Lf; $p>0.999$ for IR+Lfx2). The significant difference was identified between the IR and IR $+\mathrm{Lf}$ groups $(\mathrm{p}=0.030)$. It has been previously established that Lf stimulated hematopoiesis [11] and recovery of leukocytes [4] in mice following irradiation.

Histological analysis revealed radiation-induced hepatic responses. The number of two - and three nuclear cells increased, and a significant number of cells with mitotic figures appeared in the IR group (Figure 3). Areas of fatty hepatosis and fibrosis were also observed in animals of this group. Previously similar data were obtained [10]. Lf administration to irradiated animals (IR+Lf and IR+Lfx2) diminished reactive changes and visually reduced fibrosis zones (Figure 3). This result indicates that Lf contributed to the overall normalization of the liver state after irradiation.

Currently, the mechanism of action of Lf after exposure to gamma-irradiation is not well clarified. The anti-radiation effect of $\mathrm{Lf}$ can be based on its immunomodulatory function, the ability to suppress reactions that lead to the formation of reactive oxygen species, inhibit free radical oxidation 

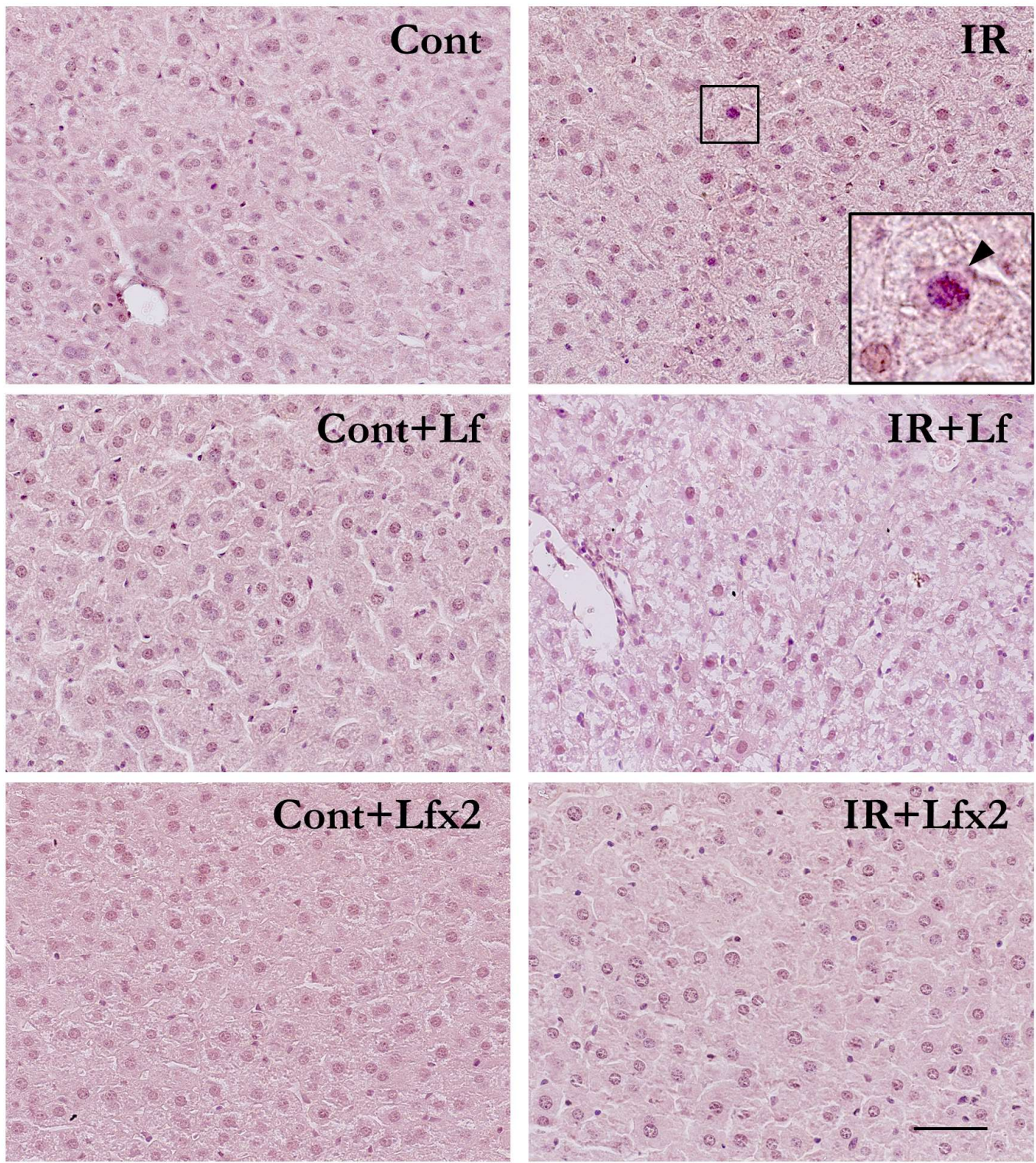

Figure 3. Effects of Lf on radiation-induced hepatic damage. H\&E staining images show liver sections from mice on day 30 after irradiation. The arrow indicates mitotic figure. Scale bar: $50 \mu \mathrm{m}$

\section{CONCLUSION}

Lf administration increased the survival rate and life span of irradiated mice during the experiment. The dynamics of changes in the body weight of these animals rapidly returned to normal. In addition, Lf had a compensatory effect on the white blood formula, serum composition and liver condition of irradiated mice. Our data suggest that Lf can reduce the negative consequences of radiation damage. The results obtained can become the basis for conducting preclinical studies of the hLf activity under the radiation exposure.

48
Acknowledgements: This work was accomplished using the resource facilities at the National Research Center «Kurchatov Institute».

\section{REFERENCES}

1. N. Orsi, "The antimicrobial activity of lactoferrin: Current status and perspectives," BioMetals, vol. 17, no. 3, pp. 189-196, Jun. 2004. https://doi.org/10.1023/B:BIOM.0000027691.86757.e $\underline{2}$ 
M. Yu. Kopaeva et al., Radioprotective effect of human lactoferrin..., RAD Conf. Proc., vol. 4, 2020, 45-49

2. I. A. García-Montoya, T. S. Cendón, S. ArévaloGallegos, and Q. Rascón-Cruz, "Lactoferrin a multiple bioactive protein: An overview," Biochim. Biophys. Acta $B B A$ - Gen. Subj., vol. 1820, no. 3, pp. 226-236, Mar. 2012. https://doi.org/10.1016/j.bbagen.2011.06.018

3. Y. Nishimura, S. Homma-Takeda, H.-S. Kim, and I. Kakuta, "Radioprotection of mice by lactoferrin against irradiation with sublethal X-rays," J. Radiat. Res. (Tokyo), vol. 55, no. 2, pp. 277-282, Mar. 2014. https://doi.org/10.1093/jrr/rrt117

4. L. Feng, J. Li, L. Qin, D. Guo, H. Ding, and D. Deng, "Radioprotective effect of lactoferrin in mice exposed to sublethal X- ray irradiation," Exp. Ther. Med., Aug. 2018. https://doi.org/10.3892/etm.2018.6570

5. N. Faraji, Y. Zhang, and A. K. Ray, "Determination of adsorption isotherm parameters for minor whey proteins by gradient elution preparative liquid chromatography," J. Chromatogr. A, vol. 1412, pp. 67-74, Sep. 2015. https://doi.org/10.1016/i.chroma.2015.08.004

6. V. Kumar, Md. I. Hassan, T. Kashav, T. P. Singh, and S. Yadav, "Heparin-binding proteins of human seminal plasma: purification and characterization," Mol. Reprod. Dev., vol. 75, no. 12, pp. 1767-1774, Dec. 2008. https://doi.org/10.1002/mrd.20910

7. M. Yu. Kopaeva, A. B. Cherepov, I. Yu. Zarayskaya, and M. V. Nesterenko, "Transport of Human Lactoferrin into Mouse Brain: Administration Routes and Distribution," Bull. Exp. Biol. Med., vol. 167, no. 4, pp. 561-567, Aug. 2019. https://doi.org/10.1007/s10517-019-04572-3
8. M. Yu. Kopaeva, I. B. Alchinova, M. V. Nesterenko, A. B. Cherepov, I. Yu. Zarayskaya, and M. Yu. Karganov, "Lactoferrin beneficially influences the recovery of physiological and behavioral indexes in mice exposed to acute gamma-irradiation," NauchnoPrakt. Zhurnal «Patogenez» [Pathogenesis], vol. 18, no. 1, pp. 29-33, Mar. 2020. https://doi.org/10.25557/2310-0435.2020.01.29-33

9. M. Karganov, I. Alchinova, E. Arkhipova, and A. V. Skalny, "Laser Correlation Spectroscopy: Nutritional, Ecological and Toxic Aspects," Biophysics ed. A N Misra (InTech), pp. 1-16, 2012. ISBN 978-95351-0376-9 https://doi.org/10.5772/35254

10. I. Alchinova, E. Arkhipova, Yu. Medvedeva, A. Cherepov, A. Antipov, N. Lysenko, L. Noskin, and M. Karganov, "The Complex of Tests for the Quantitative Evaluation of the Effects of Radiation on Laboratory Animals," Am. J. Life Sci., vol. 3, no. 1, pp. 5-12, 2015. https://doi.org/10.11648/j.ajls.s.2015030102.12

11. A. A. Ivanov, A. M. Ulanova, N. M. Stavrakova, Iu. B. Deshevor̆, T. A. Nasonova, A. N. Koterov, K. K. Gutsenko, V. N. Mal'tsev, ["Antiradiation effects of Lactoferrin"]. Radiatsionnaia biologiia. Radioecologiia [Radiation biology. Radioecology], vol. 49, no. 4, pp. 456-461, 2009. (in Russian)

12. I. B. Alchinova, M. V. Polyakova, E. N. Yakovenko, Y. S. Medvedeva, I. N. Saburina, and M. Y. Karganov, "Effect of Extracellular Vesicles Formed by Multipotent Mesenchymal Stromal Cells on Irradiated Animals," Bull. Exp. Biol. Med., vol. 166, no. 4, pp. 574-579, 2019. https://doi.org/10.1007/s10517-019-04394-3 